\title{
Maternal vitamin D concentrations are associated with faster childhood reaction time and response speed, but not with motor fluency and flexibility, at the age of 5-6 years: the Amsterdam Born Children and their Development $(A B C D)$ Study
}

\author{
Elske M. Brouwer-Brolsma ${ }^{1 *}$, Tanja G. M. Vrijkotte ${ }^{2}$ and Edith J. M. Feskens ${ }^{1}$ \\ ${ }^{1}$ Division of Human Nutrition, Wageningen University, 6700 AA Wageningen, The Netherlands \\ ${ }^{2}$ Department of Public Health, Amsterdam Public Health Research Institute, Academic Medical Center - University of \\ Amsterdam, 1100 DD Amsterdam, The Netherlands \\ (Submitted 5 October 2017 - Final revision received 31 March 2018 - Accepted 16 April 2018 - First published online 30 May 2018)
}

\section{Abstract}

About $57 \%$ of the pregnant European women have 25-hydroxyvitamin D (25(OH)D) concentrations below 50 nmol/1. However, as data on the impact of gestational vitamin D deficiency on maternal and fetal health are limited, the WHO does not advocate vitamin D supplementation as part of routine antenatal care. We explored associations between first trimester maternal 25(OH)D status and childhood cognition at 5-6 years of age ( $n$ 1854, primarily Caucasian). Median serum 25(OH)D was determined at 13 (interquartile range 12-14) weeks of gestation. Childhood attention, motor fluency and flexibility and executive function were assessed using the Amsterdam Neuropsychological Tasks. Restricted cubic splines and linear regression analyses were used to analyse the data while adjusting for many maternal and child related covariates. Higher 25(OH)D status (nmol/l) was associated with better attention and executive functioning as shown by a faster reaction time $(\beta-0.30$ (sD 0.14$) \mathrm{ms}, P=0.03$ ), faster response speed $(\beta-0.58$ (sD 0.21$) \mathrm{ms}, P=0.006$ ), and better response speed stability $(\beta-0.45$ (sD 0.17$) \mathrm{ms}, P=0.009)$. No associations were observed of serum $25(\mathrm{OH}) \mathrm{D}$ with motor fluency and flexibility. Associations were most pronounced among children of African origin ( $n$ 205) as compared with those of Caucasian or another origin, for example attention (reaction time, $\beta-2.06(\mathrm{sD} 0.70) \mathrm{ms}, P=0.004)$ and executive function (response speed, $\beta-1.95$ (sD 0.94$) \mathrm{ms}, P=0.04$ ). Concluding, maternal 25(OH)D status was significantly associated with childhood attention and executive function, while no associations were observed for $25(\mathrm{OH}) \mathrm{D}$ status with motor fluency and flexibility.

Key words: 25-Hydroxyvitamin D: Cognition: Maternal nutrition: Prenatal nutrition: Offspring

Vitamin D deficiency is known to be a common problem among a variety of population groups, including pregnant women $^{(1)}$ and dark-skinned people ${ }^{(2)}$. Several studies suggest that an adequate vitamin $\mathrm{D}$ status may prevent pregnancy complications and beneficially affect maternal and fetal health $^{(3,4)}$. However, as the scientific evidence on this matter is still scarce, the WHO does not recommend vitamin D supplementation in pregnancy as part of routine antenatal care ${ }^{(5)}$.

As the fetus is completely dependent on the vitamin D stores of the mother, low maternal vitamin $\mathrm{D}$ concentrations may amongst others influence the developing child by affecting fetal brain development and function (reviewed in Pet et al. ${ }^{(6)}$ ). Namely, ex vivo studies have revealed the presence of vitamin D receptors in many body tissues, including the placenta ${ }^{(7)}$ and the brain $^{(8)}$. Moreover, rodent studies have shown effects of low maternal vitamin D concentrations on offspring brain morpho$\operatorname{logy}{ }^{(9,10)}$, brain physiology ${ }^{(11,12)}$, as well as behaviour ${ }^{(13-15)}$.
Human observational studies on their turn have demonstrated subtle associations between maternal vitamin D status and cognitive function in the child as well, predominantly in Caucasian populations ${ }^{(16-21)}$. Significant positive associations were reported between first/second trimester maternal serum 25-hydroxyvitamin D (25(OH)D) concentrations and mental/ psychomotor scores in 14-month-old Spanish toddlers $(n \text { 1820) })^{(18)}$, second trimester 25(OH)D status and language impairment in 5- and 10-year-old Australian children ( $n 534$ and $474)^{(19)}$, and third trimester $25(\mathrm{OH}) \mathrm{D}$ status and language scores in 6-month-old Vietnamese infants $(n 960)^{(16)}$. In contrast, no associations were observed of second trimester maternal 25 $(\mathrm{OH}) \mathrm{D}$ status and cord blood 25(OH)D with mental scores among 8-month-old American infants $\left(n\right.$ 3825) ${ }^{(17)}$. Moreover, second or third trimester $25(\mathrm{OH}) \mathrm{D}$ concentrations were not associated with total intelligence quotient (IQ) scores, tests of scholar achievement, reading and spelling, language impairments

Abbreviations: 25(OH)D, 25-hydroxyvitamin D; ABCD, Amsterdam Born Children and their Development.

* Corresponding author: E. M. Brouwer-Brolsma, fax +31 317 484987, email elske.brouwer-brolsma@wur.nl 
or verbal IQ among Danish $(n \text { 798 })^{(21)}$, English $(n \text { 178) })^{(20)}$ and American children $(n \text { 2987 })^{(17)}$. The discrepancy between study outcomes may be partly explained by the large heterogeneity between studies, for example differences in timing of blood sampling (1st, 2nd or 3rd trimester), mean maternal $25(\mathrm{OH}) \mathrm{D}$ concentrations, cognitive testing procedures, the age of cognitive testing, included covariates and ethnicity. Most studies explored associations of maternal 25(OH)D status in mid- and late gestation with childhood cognition. However, the human brain already starts to develop in the 3rd gestational week where the basic brain structures and central nervous system are already formed after 8 weeks of pregnancy ${ }^{(22)}$. Therefore, studies on the potential role of early gestation $25(\mathrm{OH}) \mathrm{D}$ concentrations in childhood cognition are needed as well.

Thus, the results of human studies are inconclusive and data on the role of early gestation $25(\mathrm{OH}) \mathrm{D}$ concentrations in relation to childhood cognition are limited. With this study we aimed to contribute to the current evidence by investigating associations between first trimester maternal 25(OH)D concentrations and child cognitive performance at age 5-6 years in the Dutch multi-ethnic Amsterdam Born Children and their Development (ABCD) mother-child cohort, expecting a better cognitive performance among those exposed to higher intrauterine $25(\mathrm{OH}) \mathrm{D}$ concentrations. As $25(\mathrm{OH}) \mathrm{D}$ concentrations were expected to be lower in participants with darker skin colour - leading to the hypothesis that potential associations may be more pronounced in specific ethnic groups - we also conducted stratified analyses based on ethnic background.

\section{Methods}

\section{Participants}

This prospective study was conducted using data from the ABCD study, a population-based, multi-ethnic mother-child cohort. The design of the ABCD study has been extensively described by van Eijsden et $a l .{ }^{(23)}$. Women were recruited, between January 2003 and March 2004, during their first antenatal visit to the general practitioner, midwife or gynaecologist. The ABCD study has been approved by the medical ethical committees of the participating hospitals and the Registration Committee of Amsterdam. All mothers gave written informed consent.

\section{Population for analyses}

In total, 12373 pregnant inhabitants of Amsterdam were invited to participate in the study; 8267 women filled out a pregnancy questionnaire; 7863 live-born singleton infants and 132 live-born multiples were delivered. Blood samples were obtained of 4389 women at the first prenatal visit (median 13 weeks gestational age, interquartile range (IQR) 12-14), where $25(\mathrm{OH}) \mathrm{D}$ status was determined in 4250 singleton mother-child pairs. A period of 5 years after birth, 6161 motherchild pairs were invited for follow-up measurements. Questionnaires were returned for 4488 pairs of whom 3132 participated in cognitive testing. Data on both $25(\mathrm{OH}) \mathrm{D}$ status and cognitive measurements at 5-6 years was available of 1854 participants. Fig. 1 provides a schematic overview of the participant flow.

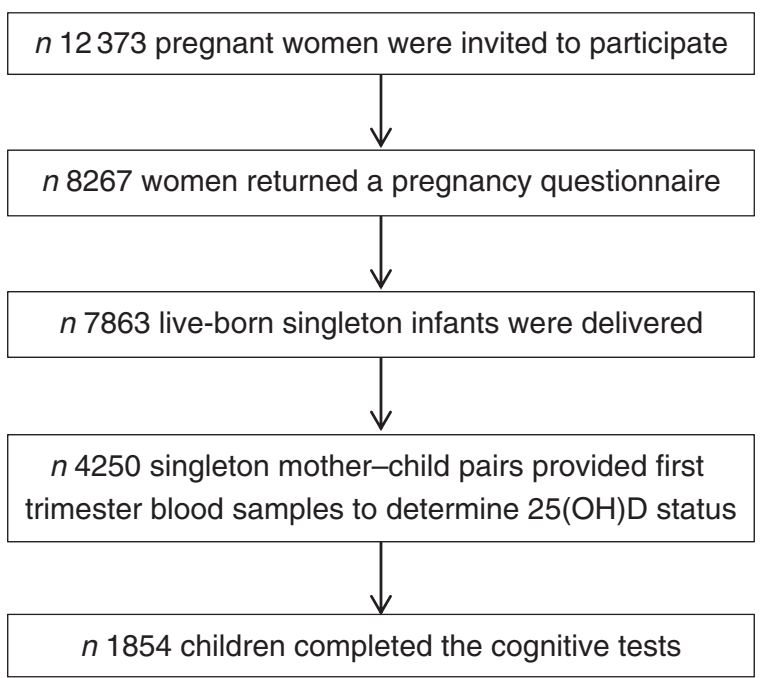

Fig. 1. Participant flow chart of the Amsterdam Born Children and their Development study investigating associations between first trimester 25hydroxyvitamin $\mathrm{D}(25(\mathrm{OH}) \mathrm{D})$ status and childhood cognition at age 5-6 years.

\section{Vitamin D status}

First trimester 25(OH)D concentrations were determined by enzyme immunoassay (OCTEIA AC-57F1; IDS Ltd) at the Regional Laboratory of Amsterdam. Intra-assay and inter-assay coefficients were 8 and $<10 \%$, respectively ${ }^{(24)}$. Data indicate that the average $25(\mathrm{OH}) \mathrm{D}$ concentrations measured with the OCTEIA IDS are approximately -13.7 (SD 1.6) nmol/1 lower than the Vitamin D External Quality Assessment Scheme mean $(P<0.0001)$ with a negative difference that becomes larger with increasing $25(\mathrm{OH}) \mathrm{D}$ concentrations ${ }^{(25)}$.

\section{Cognitive performance}

Domain-specific cognitive performance - including attention, motor fluency and flexibility, and executive function - was assessed by means of computerised tasks of the Amsterdam Neuropsychological Tasks (ANT). The ANT has been extensively used and illustrated elsewhere ${ }^{(26-29)}$ and judged acceptable, which is for instance demonstrated by strong effect sizes in the expected direction for the executive function task ${ }^{(29)}$. Besides, the ANT has been shown to have satisfactory psychometric properties as shown by test-retest correlations ranging from 0.70 to 0.85 (summarised in Koekkoek et al. ${ }^{(30)}$ ). Cognitive measurements were conducted in the morning or early afternoon by well-trained researchers according to conscientious procedures. In short, children were individually tested in a quiet room where they first received a verbal task instruction while simultaneously showing an example of the task on the laptop screen. Subsequently, the child completed a practice run to become familiar with the task stimuli and response mode. The actual test trial started once the researcher ascertained that the child understood the task. The cognitive test battery included four tasks, specifically 'baseline speed' to measure attention, 'pursuit' and 'tracking' to measure motor fluency and flexibility, and 'response organisation objects' to measure executive function (inherent response inhibition and response flexibility). A more extensive description of these tests can be found in the paper by Finken et al. ${ }^{(31)}$. 


\section{Covariates}

Information on age (years) and sex of child (boy/girl, $n(\%)$ ), birth order (first/second/third/fourth/fifth or more, $n$ (\%)), birth weight $(\mathrm{g})$, gestational age at birth (weeks), ethnicity based on birth country of the grandmother of the child categorised into three groups ((1) Caucasian: Dutch and other Caucasian populations; (2) African: Creole Surinamese, Antillean and Ghanaian; (3) other: Hindu, Turkish, Moroccan and other nonCaucasian), $n(\%))$, maternal education years after primary school $(<6 / 6-10 />10$ years, $n(\%))$, breast-feeding duration (weeks), use of vitamin D drops in infancy (no/yes), game-time child at age 5-6 $(<1 / \geq 1 \mathrm{~h}, n(\%))$, television-time child at age 5-6 $(<1 / \geq 1 \mathrm{~h}, n(\%))$, maternal age (years), smoking during pregnancy (yes/no, $n(\%)$ ), alcohol consumption during pregnancy (yes/no, $n$ (\%)) and physical activity level during pregnancy (no, low, moderate, vigorous $(n, \%)$ ) was collected by means of questionnaires completed by the mothers during pregnancy, 3 months after delivery and when children were 5-6 years old. Pre-pregnancy BMI (weight/height ${ }^{2}$ ) was calculated using measures of self-reported height and weight. Winter/summer season was defined based on the date of blood sampling. Blood samples collected in June, July, August, September, October and November were defined as 'summer' months; samples collected in December, January, February, March, April and May were defined as 'winter' months ${ }^{(32)}$.

\section{Statistical analyses}

Participant characteristics are reported over maternal serum $25(\mathrm{OH}) \mathrm{D}$ quartiles as means and standard deviations, or numbers and percentages. Moreover, columns displaying the characteristics of the whole ABCD study population as well as the total subsample were included. Medians and IQR were used to report skewed variables. Differences between quartiles were analysed by means of ANOVA (normally distributed continuous variables), Kruskal-Wallis (skewed continuous variables) and $\chi^{2}$ test (categorical variables). Linearity of the associations between maternal vitamin D status and childhood cognitive performance was investigated using restricted cubic spline regression, which was conducted with three knots located at the 1st, 5th and 9th deciles of $25(\mathrm{OH}) \mathrm{D}$ status. Multivariable regression analyses were used to quantify the strength of the associations between maternal 25(OH)D status and child cognitive performance at age 5 years. Analyses were adjusted for age, sex of child, date of blood sampling using a sinusoidal function $^{(33)}$ (model 1), birth order, birth weight, gestational age at birth, ethnicity, maternal education years after primary school, breast-feeding duration, vitamin D drops, game-time, television-time, maternal age, pre-pregnancy BMI, smoking during pregnancy, alcohol consumption during pregnancy and physical activity level during pregnancy (model 2). Potential interactions of maternal 25(OH)D status with ethnicity, sex and age were analysed by including a cross-product term in the fully adjusted model, and by analysing the data per ethnic group (i.e. Caucasian, African, other), sex (i.e. boy, girl) and age-group (i.e. $<5.6$ years, $\geq 5.6$ years). A two-sided $P$ value of $\leq 0.05$ was considered to be statistically significant. Except for the restricted cubic spline analyses, all statistical analyses were performed using the statistical package SAS, version 9.3 (SAS Institute Inc.). Restricted cubic spline analyses were performed using $\mathrm{R}$ version 2.15 .

\section{Results}

Participant characteristics stratified by serum 25(OH)D quartile are shown in Table 1 . In this population, the mean $25(\mathrm{OH}) \mathrm{D}$ concentration was 60 (sD 31) nmol/l of which 38 and $18 \%$ of the women had a status below 50 and $30 \mathrm{nmol} / \mathrm{l}$, respectively. Compared with women with higher $25(\mathrm{OH}) \mathrm{D}$ concentrations, women exhibiting the lowest $25(\mathrm{OH}) \mathrm{D}$ concentrations were significantly younger, more likely to smoke during their pregnancy, had a higher pre-pregnancy BMI and less education years. Women with the lowest $25(\mathrm{OH}) \mathrm{D}$ concentrations were also less likely to consume alcohol during their pregnancy and to physically exercise. Children exposed to the lowest intrauterine $25(\mathrm{OH}) \mathrm{D}$ concentrations had a significantly lower birth weight, were less likely to be the first child in the family, had a shorter breast-feeding duration, and were less likely to have received vitamin D drops/supplements after birth compared with children exposed to higher intra-uterine $25(\mathrm{OH}) \mathrm{D}$ concentrations. Whereas $87-98 \%$ of the mother-child pairs in the second, third, or fourth $25(\mathrm{OH}) \mathrm{D}$ quartile were of Caucasian origin, only $44 \%$ of the mother-child pairs in the first $25(\mathrm{OH}) \mathrm{D}$ quartile were Caucasian. Moreover, compared with the total ABCD-study sample, the subsample used for the analyses on $25(\mathrm{OH}) \mathrm{D}$ status and childhood cognition was somewhat higher educated, more likely to be exposed to alcohol during pregnancy and vitamin $\mathrm{D}$ drops after birth, and more likely to be Caucasian. Crude analyses furthermore suggest poorer motor fluency and flexibility with higher 25(OH)D concentrations as shown by, for example, a larger mean distance ( $\mathrm{mm}$ ) between mouse cursor and the actual target on the pursuit for children exposed to higher intra-uterine $25(\mathrm{OH}) \mathrm{D}$ concentrations (online Supplementary Table S1).

Restricted cubic splines for first trimester serum 25(OH)D status and childhood cognitive function that were adjusted for all available covariates suggested linearity (online Supplementary Fig. S1). In unadjusted linear regression analyses, the associations of serum $25(\mathrm{OH}) \mathrm{D}$ with motor fluency and flexibility, represented by the tasks pursuit and tracking, reached statistical significance. After adjustment for age, sex of the child and date of blood sampling, these associations attenuated and became non-significant. In contrast, adjustment for age, sex of the child and date of blood sampling, substantially strengthened the associations between serum $25(\mathrm{OH}) \mathrm{D}$ and the cognitive domains attention (i.e. baseline speed) and executive function (i.e. response organisation objects part 1). After adjustment for all available covariates the associations remained, showing a significantly faster reaction time, faster response speed and better response speed stability per $1 \mathrm{nmol} / 1$ increase in 25(OH)D, $\beta-0.30$ (sD 0.14), $\beta-0.58$ (sD $0 \cdot 21$ ) and $\beta-0.45$ (sD $0 \cdot 17$ ), respectively (Table 2 ). Additional adjustment for the number of errors somewhat attenuated the associations for response organisation objects part 1 , showing $\beta-0.49$ (SD 0.19) $(P=0.01)$ for response speed and $\beta-0.38$ 
Table 1. Amsterdam Born Children and their Development (ABCD)-study population characteristics by quartiles $(\mathrm{Q})$ of maternal 25-hydroxyvitamin $D$ status (nmol/l)* (Numbers and percentages; mean values and standard deviations)

\begin{tabular}{|c|c|c|c|c|c|c|c|c|c|c|c|c|c|}
\hline & \multicolumn{2}{|c|}{$\begin{array}{l}\text { Total ABCD population } \\
(n \text { 8267)† }\end{array}$} & \multicolumn{2}{|c|}{$\begin{array}{l}\text { Population for analyses } \\
\qquad(n \text { 1854 })^{\star}\end{array}$} & \multicolumn{2}{|c|}{$\begin{array}{l}\mathrm{Q} 1(<37) \\
(n \text { 463) }\end{array}$} & \multicolumn{2}{|c|}{$\begin{array}{l}\text { Q2 (37-60) } \\
\quad(n 462)\end{array}$} & \multicolumn{2}{|c|}{$\begin{array}{l}\text { Q3 (61-80) } \\
\quad(n 464)\end{array}$} & \multicolumn{2}{|c|}{$\begin{array}{l}\text { Q4 }(>80) \\
(n \text { 464) }\end{array}$} & \multirow[b]{2}{*}{$P$} \\
\hline & $n$ & $\%$ & $n$ & $\%$ & $n$ & $\%$ & $n$ & $\%$ & $n$ & $\%$ & $n$ & $\%$ & \\
\hline \multicolumn{14}{|l|}{ Maternal characteristics } \\
\hline $25(\mathrm{OH}) \mathrm{D}(\mathrm{nmol} / \mathrm{l})$ & & & & & & & & & & & & & $<0.0001$ \\
\hline $\begin{array}{l}\text { Mean } \\
\text { SD }\end{array}$ & \multicolumn{2}{|c|}{$\begin{array}{l}56 \\
31\end{array}$} & \multicolumn{2}{|c|}{$\begin{array}{l}60 \\
31\end{array}$} & \multicolumn{2}{|c|}{$\begin{array}{c}24 \\
8\end{array}$} & \multicolumn{2}{|c|}{$\begin{array}{c}49 \\
6\end{array}$} & & & \multicolumn{2}{|c|}{$\begin{array}{l}100 \\
22\end{array}$} & \\
\hline Winter blood sample & 2405 & 57 & 1007 & 55 & 346 & 75 & 298 & 65 & 208 & 45 & 155 & 33 & $<0.0001$ \\
\hline Age (years) & & & & & & & & & & & & & $<0.0001$ \\
\hline Mean & \multicolumn{2}{|c|}{$\begin{array}{c}30 \cdot 8 \\
5.2\end{array}$} & \multirow{2}{*}{\multicolumn{2}{|c|}{$\begin{array}{c}31.9 \\
4.4\end{array}$}} & \multicolumn{2}{|c|}{$30 \cdot 2$} & \multicolumn{2}{|c|}{$32 \cdot 3$} & \multicolumn{2}{|c|}{$32 \cdot 4$} & \multicolumn{2}{|c|}{$32 \cdot 6$} & \\
\hline $\begin{array}{l}\mathrm{SD} \\
\text { Pre-pregnancy BMI }\left(\mathrm{kg} / \mathrm{m}^{2}\right)\end{array}$ & & & & & & & & & & & & & $<0.0001$ \\
\hline Mean & & & & & & & & & & & & & $<0.0001$ \\
\hline Smokers & 778 & 9 & 154 & 8 & 48 & 10 & 47 & 10 & 29 & 6 & 30 & 6 & 0.03 \\
\hline Alcohol consumption & 1704 & 21 & 553 & 30 & 64 & 14 & 132 & 29 & 178 & 38 & 179 & 39 & $<0.0001$ \\
\hline $\begin{array}{l}\text { Education years after primary school } \\
<6\end{array}$ & & & & & & & & & & & & & \\
\hline$<6$ & 1966 & 24 & 238 & 13 & 141 & 30 & 51 & 11 & 23 & 5 & 23 & 5 & $<0.0001$ \\
\hline $6-10$ & 3133 & 38 & 654 & 35 & 189 & 41 & 156 & 34 & 147 & 32 & 162 & 35 & \\
\hline$>10$ & 3168 & 38 & 962 & 52 & 134 & 29 & 255 & 55 & 294 & 63 & 279 & 60 & \\
\hline Physical activity & & & & & & & & & & & & & \\
\hline No & 1503 & 18 & 204 & 11 & 112 & 24 & 51 & 11 & 26 & 6 & 15 & 3 & $<0.0001$ \\
\hline Low & 3486 & 42 & 659 & 36 & 208 & 45 & 165 & 36 & 153 & 33 & 133 & 29 & \\
\hline Moderate & 2645 & 32 & 787 & 42 & 125 & 27 & 187 & 40 & 236 & 51 & 239 & 52 & \\
\hline Vigorous & 625 & 8 & 202 & 11 & 17 & 4 & 59 & 13 & 49 & 10 & 77 & 17 & \\
\hline Child characteristics & & & & & & & & & & & & & \\
\hline Gestational age at birth (weeks) & & & & & & & & & & & & & 0.24 \\
\hline Mean & & & & & & & & & & & & & \\
\hline Mean & & & & & & & & & & & & & $0.000 /$ \\
\hline SD & & & & & & & & & & & & & \\
\hline Birth order & & & & & & & & & & & & & \\
\hline First & 3356 & 40 & 802 & 43 & 162 & 35 & 210 & 45 & 225 & 49 & 205 & 44 & $<0.0001$ \\
\hline Second & 2539 & 31 & 581 & 31 & 139 & 30 & 149 & 32 & 137 & 30 & 156 & 34 & \\
\hline Third & 1306 & 16 & 286 & 15 & 85 & 18 & 66 & 14 & 67 & 14 & 68 & 15 & \\
\hline Fourth & 563 & 7 & 101 & 6 & 36 & 8 & 21 & 5 & 20 & 4 & 24 & 5 & \\
\hline Fifth or later & 501 & 6 & 83 & 5 & 41 & 9 & 16 & 4 & 15 & 3 & 11 & 2 & \\
\hline Duration of breast-feeding (weeks) & & & & & & & & & & & & & 0.006 \\
\hline Mean & & & & & & & & & & & & & \\
\hline & & & & & & & & & & & & & \\
\hline Vitamin D drops & 4126 & 50 & 1382 & 75 & 295 & 64 & 345 & 75 & 387 & 83 & 355 & 77 & $<0.0001$ \\
\hline Age at assessment (years) & & & & & & & & & & & & & $<0.0001$ \\
\hline Mean & & & & & & & & & & & & & \\
\hline Boy & 1555 & 50 & 901 & 49 & 220 & 47 & 240 & 52 & 212 & 46 & 229 & 49 & 0.26 \\
\hline$>1 \mathrm{~h} / \mathrm{d}$ gaming & 262 & 7 & 97 & 6 & 57 & 14 & 15 & 4 & 13 & 3 & 12 & 3 & $<0.0001$ \\
\hline$>1 \mathrm{~h} / \mathrm{d}$ television & 2123 & 54 & 912 & 53 & 287 & 70 & 218 & 51 & 203 & 46 & 204 & 46 & $<0.0001$ \\
\hline Ethnicity & & & & & & & & & & & & & \\
\hline Caucasian & 5444 & 66 & 1502 & 81 & 202 & 44 & 404 & 87 & 441 & 95 & 455 & 98 & $<0.0001$ \\
\hline African & 2163 & 26 & 275 & 15 & 214 & 46 & 36 & 8 & 17 & 4 & 8 & 2 & \\
\hline Other & 646 & 8 & 77 & 4 & 48 & 10 & 22 & 5 & 6 & 1 & 1 & 0 & \\
\hline
\end{tabular}

* Differences between quartiles were analysed by means of ANOVA (normally distributed continuous variables), Kruskal-Wallis (skewed continuous variables), or $x^{2}$ test (categorical variables). Missing's for the population for analyses: pre-pregnancy BMI, $n$ 111; alcohol during pregnancy, $n$ 1; physical activity, $n$ 1; gestational age, $n$ 4; birth weight, $n$ 10; birth order, $n$ 1; duration of breast-feeding, $n 22$; gaming, $n 144$; television, $n 136$.

† Data are selected based on the mothers returning a pregnancy questionnaire ( $n$ 8267); within this 'total ABCD population', vitamin D status was determined in $n 4250$. Missing for the population returning the pregnancy questionnaire: pre-pregnancy BMI, $n$ 784; alcohol during pregnancy, $n 4$; smoking during pregnancy, $n$ 12; physical activity, $n$ 7; gestational age, $n 394$; birth weight, $n 475$; birth order, $n$ 1; duration of breast-feeding, $n 2109$; age at assessment, $n$ 5156; sex, $n$ 5156; gaming, $n$ 4348; television, $n$ 4335; ethnicity, $n 13$. 
Table 2. Linear regression analyses showing the associations between 25-hydroxyvitamin D status and child cognition at age 5-6 years ( $\beta$-Coefficients and standard deviations)

\begin{tabular}{|c|c|c|c|c|c|c|c|c|c|}
\hline & \multicolumn{3}{|c|}{ Crude model } & \multicolumn{3}{|c|}{ Model $1^{*}$} & \multicolumn{3}{|c|}{ Model $2 \dagger$} \\
\hline & $\beta$ & SD & $P$ & $\beta$ & SD & $P$ & $\beta$ & SD & $P$ \\
\hline \multicolumn{10}{|l|}{ Attention } \\
\hline \multicolumn{10}{|l|}{ Baseline speed } \\
\hline Reaction time (ms) & -0.02 & 0.11 & 0.89 & -0.42 & 0.11 & 0.0001 & -0.30 & 0.14 & 0.03 \\
\hline Response speed stability (ms) & 0.009 & 0.11 & 0.93 & -0.30 & 0.11 & 0.008 & -0.09 & 0.14 & 0.53 \\
\hline \multicolumn{10}{|l|}{ Motor fluency and flexibility } \\
\hline \multicolumn{10}{|l|}{ Pursuit } \\
\hline Movement accuracy (mm) & 0.01 & 0.00 & 0.02 & 0.00 & 0.01 & 0.92 & 0.00 & 0.01 & 0.85 \\
\hline Accuracy stability $(\mathrm{mm})$ & 0.01 & 0.00 & 0.01 & 0.00 & 0.00 & 0.94 & 0.00 & 0.01 & 0.75 \\
\hline \multicolumn{10}{|l|}{ Tracking } \\
\hline Movement accuracy (mm) & 0.00 & 0.00 & 0.07 & 0.00 & 0.00 & 0.15 & 0.00 & 0.00 & 0.68 \\
\hline Accuracy stability $(\mathrm{mm})$ & 0.00 & 0.00 & 0.08 & 0.00 & 0.00 & 0.72 & 0.00 & 0.00 & 0.37 \\
\hline Movement speed (s) & 0.01 & 0.01 & 0.02 & 0.00 & 0.01 & 0.83 & -0.01 & 0.01 & 0.40 \\
\hline \multicolumn{10}{|l|}{ Executive function } \\
\hline \multicolumn{10}{|l|}{ Response organisation objects } \\
\hline Response speed part 1 (ms) & -0.03 & 0.17 & 0.85 & -0.55 & 0.17 & 0.001 & -0.58 & 0.21 & 0.006 \\
\hline Response speed stability part 1 (sD, ms) & -0.11 & 0.13 & 0.40 & -0.51 & 0.14 & 0.0003 & -0.45 & 0.17 & 0.009 \\
\hline Response speed part 2 (ms) & 0.47 & 0.23 & 0.04 & -0.25 & 0.23 & 0.29 & -0.23 & 0.29 & 0.43 \\
\hline Response speed stability part 2 (sD, ms) & 0.01 & 0.18 & 0.96 & -0.47 & 0.19 & 0.01 & -0.22 & 0.23 & 0.35 \\
\hline
\end{tabular}

* Model 1 is adjusted for age, sex of child and date of blood sampling.

† Model 2 is adjusted for model 1, demographics and child's characteristics (i.e. birth order, birth weight, gestational age at birth, ethnicity (Caucasian, African, other), maternal education years after primary school $(<6,6-10,>10)$, breast-feeding duration (weeks), vitamin $\mathrm{D}$ drops (no/yes), game-time $(<1 \mathrm{~h} / \geq 1 \mathrm{~h})$, television-time $(<1 \mathrm{~h} / \geq 1 \mathrm{~h})$ and maternal characteristics (i.e. age, pre-pregnancy BMl, smoking during pregnancy (yes/no), alcohol consumption during pregnancy (yes/no) and physical activity level during pregnancy (no, low, moderate, vigorous).

Table 3. Linear regression analyses showing associations between 25 -hydroxyvitamin D status and cognitive performance at age 5-6 years stratified by ethnicity*

( $\beta$-Coefficients and standard deviations)

\begin{tabular}{|c|c|c|c|c|c|c|c|c|c|c|}
\hline & \multirow[b]{2}{*}{$P_{\mathrm{i}}$} & \multicolumn{3}{|c|}{ Caucasian } & \multicolumn{3}{|c|}{ African } & \multicolumn{3}{|c|}{ Other } \\
\hline & & $\beta$ & SD & $P$ & $\beta$ & SD & $P$ & $\beta$ & SD & $P$ \\
\hline \multicolumn{11}{|l|}{ Attention } \\
\hline \multicolumn{11}{|l|}{ Baseline speed ( $n$ 1307, 204 and 55) } \\
\hline Reaction time (ms) & 0.0002 & -0.17 & 0.14 & 0.23 & -2.06 & 0.70 & 0.004 & -1.07 & $2 \cdot 34$ & 0.65 \\
\hline Response speed stability (ms) & 0.0002 & 0.04 & 0.14 & 0.77 & -1.83 & 0.71 & 0.01 & -0.10 & 1.94 & 0.96 \\
\hline \multicolumn{11}{|l|}{ Motor fluency and flexibility } \\
\hline \multicolumn{11}{|l|}{ Pursuit ( $n$ 1322, 205 and 55) } \\
\hline Movement accuracy (mm) & 0.01 & 0.00 & 0.01 & 0.62 & -0.04 & 0.02 & 0.08 & 0.03 & 0.07 & 0.67 \\
\hline Accuracy stability $(\mathrm{mm})$ & 0.005 & 0.01 & 0.01 & 0.28 & -0.04 & 0.02 & 0.09 & 0.02 & 0.08 & 0.79 \\
\hline \multicolumn{11}{|l|}{ Tracking ( $n$ 1307, 203 and 53) } \\
\hline Movement accuracy (mm) & 0.99 & 0.00 & 0.00 & 0.39 & 0.00 & 0.01 & 0.85 & -0.02 & 0.04 & 0.60 \\
\hline Accuracy stability (mm) & 0.19 & 0.00 & 0.00 & 0.59 & 0.00 & 0.01 & 0.84 & -0.01 & 0.03 & 0.77 \\
\hline Movement speed (s) & 0.77 & -0.01 & 0.01 & 0.26 & 0.01 & 0.03 & 0.68 & 0.14 & 0.13 & 0.28 \\
\hline \multicolumn{11}{|l|}{ Executive function } \\
\hline \multicolumn{11}{|c|}{ Response organisation objects ( $n 1315,203$ and 54 ) } \\
\hline Response speed part 1 (ms) & $0 \cdot 12$ & -0.52 & 0.22 & 0.02 & -1.95 & 0.94 & 0.04 & 3.51 & 2.69 & 0.20 \\
\hline Response speed stability part 1 (sD, ms) & 0.21 & -0.41 & 0.17 & 0.02 & -1.04 & 0.89 & 0.25 & $2 \cdot 39$ & $2 \cdot 19$ & 0.28 \\
\hline Response speed part 2 (ms) & 0.59 & -0.20 & 0.31 & 0.52 & $-1 \cdot 30$ & 1.40 & 0.35 & -0.15 & 3.35 & 0.96 \\
\hline Response speed stability part 2 (sD, ms) & 0.16 & -0.12 & 0.24 & 0.61 & -1.90 & $1 \cdot 19$ & 0.11 & -3.61 & 1.85 & 0.06 \\
\hline
\end{tabular}

$P_{\mathrm{i}}, P$ for interaction.

* The models are adjusted for age and sex of child, date of blood sampling, demographics and child's characteristics (i.e. birth order, birth weight, gestational age at birth, maternal education years after primary school $(<6,6-10,>10)$, breast-feeding duration (weeks), vitamin $\mathrm{D}$ drops (no/yes), game-time $(<1 \mathrm{~h} / \geq 1 \mathrm{~h})$, television time $(<1 \mathrm{~h} / \geq 1 \mathrm{~h}))$, maternal characteristics (i.e. age, pre-pregnancy BMI, smoking during pregnancy (yes/no), alcohol consumption during pregnancy (yes/no), physical activity level during pregnancy (no, low, moderate, vigorous).

(sD 0.16) $(P=0.02)$ for response speed stability (data not shown in tables).

Associations between first trimester 25(OH)D concentrations and cognitive performance at age 5-6 years were most pronounced in mother-child pairs of African origin (Table 3).
Specifically, each nmol/1 increase in $25(\mathrm{OH}) \mathrm{D}$ status was associated with a -1.95 (SD 0.94) $\mathrm{ms}$ faster response speed in participants of African origin, whereas this was only -0.52 (sD 0.22$)$ ms among those with Caucasian origin $\left(P_{\text {for interaction: }}\right.$ : $0 \cdot 12$ ). Moreover, stratified analyses also suggested a significant 
association between serum 25(OH)D and attention in those of African origin (i.e. baseline speed, reaction time, $\beta-2.06$ (sD 0.70)), whereas no such association was observed in those of Caucasian origin (i.e. baseline speed, reaction time, $\beta 0 \cdot 17$

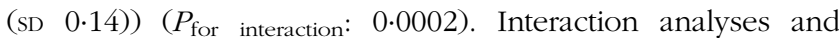
stratification for age and sex did not suggest substantial differences in the associations for these two factors (online Supplementary Table S2).

\section{Discussion}

In this Dutch multi-ethnic mother-child cohort, higher first trimester $25(\mathrm{OH}) \mathrm{D}$ status was significantly associated with a better childhood attention and executive functioning - but not motor fluency and flexibility - at age 5-6 years. Stratified analyses pointed towards more pronounced associations of first trimester $25(\mathrm{OH}) \mathrm{D}$ status with childhood attention and executive function among participants of African origin. Although statistically significant, the associations observed for the total population are very modest. For instance, for executive function a $10 \mathrm{nmol} / 1$ higher $25(\mathrm{OH}) \mathrm{D}$ status was associated with a $6-\mathrm{ms}$ faster response speed, which is only $0.6 \%$ of the average response speed on this task (i.e. $666 \mathrm{~ms}$ ). However, when looking at the associations observed in the subsample of African origin, a $10-\mathrm{nmol} / \mathrm{l}$ higher $25(\mathrm{OH}) \mathrm{D}$ status was associated with an $20 \mathrm{~ms}$ faster response speed, which is $3 \%$ of the average response time on this task in this subpopulation. Considering the low $25(\mathrm{OH}) \mathrm{D}$ concentrations in this subgroup (i.e. 29 (SD 19) nmol/l of which $87 \%$ of the women had a $25(\mathrm{OH}) \mathrm{D}$ status below $<50 \mathrm{nmol} / \mathrm{l}$ ), an average $20 \mathrm{nmol} / 1$ increase in $25(\mathrm{OH}) \mathrm{D}$ status in this subgroup would be recommended in order to meet the current recommendations as formulated by the Institute of Medicine ${ }^{(34)}$. As our data suggest that a $20-\mathrm{nmol} / 1$ increase in $25(\mathrm{OH}) \mathrm{D}$ may equal a $6 \%(40 \mathrm{~ms})$ faster response speed, we feel that this result may suggest a relevant role for maternal vitamin D status in childhood cognition among those with $25(\mathrm{OH}) \mathrm{D}$ concentrations far below the currently recommended status levels. However, as our data are observational it may be evident that future studies are warranted to confirm our findings.

To the best of our knowledge, this study belongs to one of the largest prospective cohort studies on the potential impact of maternal 25(OH)D status on childhood cognitive performance so far. Another major study in this field was conducted as part of the US Collaborative Perinatal Project (CPP), including 3146 up to 3587 mother-child pairs with data on $25(\mathrm{OH}) \mathrm{D}$ status and child cognitive development and achievement collected at the age of 8 months, and 4 and 7 years ${ }^{(17)}$. In contrast to our study, the US CPP did not point towards a potential benefit of higher $25(\mathrm{OH}) \mathrm{D}$ concentrations during gestation. Whereas the variation in $25(\mathrm{OH}) \mathrm{D}$ status in the two studies was rather similar, the US CPP used a paper-pencil IQ test to assess childhood cognition while the ABCD population was tested used computerised testing procedures. Moreover, in the US CPP, blood was predominantly (i.e. $86 \%$ ) sampled in the second trimester of gestation (median 21 (IQR 7.3) weeks), whereas blood samples in the ABCD population were obtained in the first trimester. As basic brain structures are formed in the first trimester, it may be postulated that our study points towards a specific critical window for exposure. This hypothesis is supported by previous data from the Spanish INfancia y Medio Ambiente Project ( $n$ 1820), which showed significant associations between higher early second trimester $25(\mathrm{OH}) \mathrm{D}$ concentrations and better mental and psychomotor scores. Alternatively, three somewhat smaller prospective cohort studies also showed significant associations between higher maternal or cord blood 25(OH)D concentrations and better cognitive performance, while in these studies blood was drawn at 18 weeks of gestation ${ }^{(19)}, 32$ weeks of gestation ${ }^{(16)}$ or at delivery (cord blood) ${ }^{(35)}$. Thus, these data on their turn suggest that sufficient $25(\mathrm{OH}) \mathrm{D}$ concentrations seem to be important throughout the whole pregnancy, which is also very plausible as the brain continues to develop during pregnancy where in the third trimester even the so-called brain-growth-spurt initiates. Aforementioned findings highlight the need for future studies in which $25(\mathrm{OH}) \mathrm{D}$ concentrations are measured at various time points during gestation.

Before continuing to the final conclusion of our research, first, some strengths and limitations need to be discussed. A major strength of this study relates to the multi-ethnic origin of the population, where stratified analyses showed that the link between maternal 25(OH)D status and child cognitive performance was most pronounced in those of African origin. Although the African-subsample was rather small ( $n$ 205), it may be postulated that this modification effect relates to genetic differences between the different ethnic populations. However, our data also indicate that participants of African origin had much lower 25(OH)D concentrations than Caucasian participants. Given findings of Zhu et al. ${ }^{(35)}$ and Hanieh et $a l^{(16)}$, which suggest an optimal 25(OH)D cord blood concentration somewhere between $30-50 \mathrm{nmol} / \mathrm{l}$, the modification effect observed in our study may also reflect a threshold effect of a minimally required $25(\mathrm{OH}) \mathrm{D}$ status for optimal brain development. More specifically, with a mean $25(\mathrm{OH}) \mathrm{D}$ concentration of 68 (sD 28) nmol/l in the Caucasian subsample, the 25(OH)D concentrations in the Caucasian subsample may already be optimal for fetal brain development and hence limit the possibility to detect potential adverse influences of insufficient $25(\mathrm{OH}) \mathrm{D}$ concentrations. Other strengths include the extensive neuropsychological test battery used and the possibility to adjust for many potentially relevant covariates. Conversely, limitations of our study are that not all recruited mother-child pairs participated in the cognitive assessment at age 5-6 years; thus, selection bias may have affected the observed associations. Moreover, given the sample size of our population we were only able to stratify our data according to white, dark and other skin colour based on country of birth of the grandmother, while more specific stratified analyses (i.e. differentiating between Caucasian, African, Moroccan, Turkish, Asian, etc.) would have been even more informative. In addition, using birth country of the child's grandmother as a proxy for skin colour may have led to misclassification as children of an 'African' mother, for instance, may have a Caucasian father and vice versa. Finally, although we observed an association between $25(\mathrm{OH}) \mathrm{D}$ status and executive function as measured with the response organisation object part 1, we did not observe 
an association between $25(\mathrm{OH}) \mathrm{D}$ and the response organisation object part 2. Although we do not have a definite explanation for this discrepancy, this difference may relate to the fact that the response organisation object part 2 included reversed reaction patterns (inhibition) and therefore required a more complex cognitive strategy than the response organisation object part 1 . Hence, it may be that this second part of the test was too difficult for this relatively young population and as such did not provide useful data for our analyses.

In this study we showed significant associations between low maternal vitamin D concentrations during pregnancy and a poorer attention and executive function, particularly among participants of African origin. No association was observed between $25(\mathrm{OH}) \mathrm{D}$ and motor fluency and flexibility. To substantiate this finding, further study on this potential effect is warranted, for instance by investigating vitamin $\mathrm{D}$ in relation to more robust measures of brain development and function (e.g. imaging and electroencephalography), exploring vitamin D supplementation effects on epigenetic signatures related to brain development and function, and by examining aforementioned associations in various ethnic groups that are sufficiently powered. If future studies confirm a beneficial vitamin D supplementation effect with respect to brain development and function of the developing child, the benefit of vitamin D supplementation during gestation can be easily communicated as soon as pregnant women call in for their first antenatal visit.

\section{Acknowledgements}

The authors thank all participants, principal investigators and collaborators of the ABCD study.

This study has been supported by the Netherlands Organization for Health Research and Development (ZonMw), The Hague.

E. M. B.-B. analysed the data and wrote the paper. E. J. M. F. provided input to the draft and had primary responsibility for the final content. T. G. M. V was involved in project conception, development of the overall research plan, study oversight and contributed to the finalisation of the manuscript. All authors read and approved the final manuscript.

E. M. B.-B. reports to have filed a patent related to vitamin D and cognitive executive function. T. G. M. V has nothing to disclose. E. J. M. F. reports to have filed a patent related to vitamin $\mathrm{D}$ and cognitive executive function.

\section{Supplementary material}

For supplementary material/s referred to in this article, please visit https://doi.org/10.1017/S0007114518001319

\section{References}

1. Saraf R, Morton SM, Camargo CA Jr, et al. (2015) Global summary of maternal and newborn vitamin D status - a systematic review. Matern Child Nutr 12, 647-668.

2. Yao S, Hong CC, Bandera EV, et al. (2017) Demographic, lifestyle, and genetic determinants of circulating concentrations of 25-hydroxyvitamin D and vitamin D-binding protein in African American and European American women. Am J Clin Nutr 105, 1362-1371.

3. Hypponen E, Cavadino A, Williams D, et al. (2013) Vitamin D and pre-eclampsia: original data, systematic review and meta-analysis. Ann Nutr Metab 63, 331-340.

4. Zhang MX, Pan GT, Guo JF, et al. (2015) Vitamin D deficiency increases the risk of gestational diabetes mellitus: a meta-analysis of observational studies. Nutrients $\mathbf{7}$, 8366-8375.

5. World Health Organization (2012) Guideline: Vitamin D Supplementation in Pregnant Women. Geneva: WHO.

6. Pet MA \& Brouwer-Brolsma EM (2016) The impact of maternal vitamin d status on offspring brain development and function: a systematic review. Adv Nutr 7, 665-678.

7. Adams JS \& Hewison M (2012) Extrarenal expression of the 25-hydroxyvitamin D-1-hydroxylase. Arch Biochem Biophys 523, 95-102.

8. Eyles DW, Liu PY, Josh P, et al. (2014) Intracellular distribution of the vitamin $\mathrm{D}$ receptor in the brain: comparison with classic target tissues and redistribution with development. Neuroscience 268, 1-9.

9. Eyles D, Brown J, Mackay-Sim A, et al. (2003) Vitamin $\mathrm{D}_{3}$ and brain development. Neuroscience 118, 641-653.

10. Feron F, Burne TH, Brown J, et al. (2005) Developmental Vitamin $\mathrm{D}_{3}$ deficiency alters the adult rat brain. Brain Res Bull 65, 141-148.

11. Eyles D, Almeras L, Benech P, et al. (2007) Developmental vitamin D deficiency alters the expression of genes encoding mitochondrial, cytoskeletal and synaptic proteins in the adult rat brain. J Steroid Biochem Mol Biol 103, 538-545.

12. Grecksch G, Ruthrich H, Hollt V, et al. (2009) Transient prenatal vitamin $D$ deficiency is associated with changes of synaptic plasticity in the dentate gyrus in adult rats. Psychoneuroendocrinology 34, Suppl. 1, S258-S264.

13. Eyles DW, Rogers F, Buller K, et al. (2006) Developmental vitamin D (DVD) deficiency in the rat alters adult behaviour independently of HPA function. Psychoneuroendocrinology 31, 958-964.

14. Fernandes de Abreu DA, Nivet E, Baril N, et al. (2010) Developmental vitamin D deficiency alters learning in C57Bl/ 6J mice. Behav Brain Res 208, 603-608.

15. Kesby JP, Burne TH, McGrath JJ, et al. (2006) Developmental vitamin D deficiency alters MK 801-induced hyperlocomotion in the adult rat: an animal model of schizophrenia. Biol Psychiatry 60, 591-596.

16. Hanieh S, Ha TT, Simpson JA, et al. (2014) Maternal vitamin D status and infant outcomes in rural Vietnam: a prospective cohort study. PLOS ONE 9, e99005.

17. Keim SA, Bodnar LM \& Klebanoff MA (2014) Maternal and cord blood $25(\mathrm{OH})$-vitamin D concentrations in relation to child development and behaviour. Paediatr Perinat Epidemiol 28, 434-444.

18. Morales E, Guxens M, Llop S, et al. (2012) Circulating 25-hydroxyvitamin D3 in pregnancy and infant neuropsychological development. Pediatrics 130, e913-e920.

19. Whitehouse AJ, Holt BJ, Serralha M, et al. (2012) Maternal serum vitamin D levels during pregnancy and offspring neurocognitive development. Pediatrics 129, 485-493.

20. Gale CR, Robinson SM, Harvey NC, et al. (2008) Maternal vitamin D status during pregnancy and child outcomes. Eur J Clin Nutr 62, 68-77.

21. Strom M, Halldorsson TI, Hansen S, et al. (2014) Vitamin D measured in maternal serum and offspring neurodevelopmental outcomes: a prospective study with long-term follow-up. Ann Nutr Metab 64, 254-261. 
22. Stiles J \& Jernigan TL (2010) The basics of brain development. Neuropsychol Rev 20, 327-348.

23. van Eijsden M, Vrijkotte TG, Gemke RJ, et al. (2011) Cohort profile: the Amsterdam Born Children and their Development (ABCD) study. Int J Epidemiol 40, 1176-1186.

24. Leffelaar ER, Vrijkotte TG \& van Eijsden M (2010) Maternal early pregnancy vitamin D status in relation to fetal and neonatal growth: results of the multi-ethnic Amsterdam Born Children and their Development cohort. Br J Nutr 104, 108-117.

25. Hypponen E, Turner S, Cumberland P, et al. (2007) Serum 25-hydroxyvitamin D measurement in a large population survey with statistical harmonization of assay variation to an international standard. I Clin Endocrinol Metab 92 , 4615-4622.

26. Gunther T, Herpertz-Dahlmann B \& Konrad K (2005) [Reliability of attention and verbal memory tests with normal children and adolescents - clinical implications]. $Z$ Kinder Jugendpsychiatr Psychother 33, 169-179.

27. Huijbregts S, de Sonneville L, Licht R, et al. (2002) Inhibition of prepotent responding and attentional flexibility in treated phenylketonuria. Dev Neuropsychol 22, 481-499.

28. Rowbotham I, Pit-ten Cate IM, Sonuga-Barke EJ, et al. (2009) Cognitive control in adolescents with neurofibromatosis type 1. Neuropsychology 23, 50-60.
29. De Sonneville L (2005) Amsterdam neuropsychological tasks: scientific and clinical applications. Tijdschrift voor Neuropsychologie $\mathbf{0}, 27-41$.

30. Koekkoek S, de Sonneville LM, Wolfs TF, et al. (2008) Neurocognitive function profile in HIV-infected school-age children. Eur J Paediatr Neurol 12, 290-297.

31. Finken MJ, van Eijsden M, Loomans EM, et al. (2013) Maternal hypothyroxinemia in early pregnancy predicts reduced performance in reaction time tests in 5- to 6-year-old offspring. J Clin Endocrinol Metab 98, 1417-1426.

32. Brouwer-Brolsma EM, Vaes AM, van der Zwaluw NL, et al. (2016) Relative importance of summer sun exposure, vitamin $\mathrm{D}$ intake, and genes to vitamin D status in Dutch older adults: the B-PROOF study. J Steroid Biochem Mol Biol 164, 168-176.

33. Sachs MC, Shoben A, Levin GP, et al. (2013) Estimating mean annual 25-hydroxyvitamin D concentrations from single measurements: the Multi-Ethnic Study of Atherosclerosis. Am J Clin Nutr 97, 1243-1251.

34. Ross AC, Manson JE, Abrams SA, et al. (2011) The 2011 report on dietary reference intakes for calcium and vitamin D from the Institute of Medicine: what clinicians need to know. J Clin Endocrinol Metab 96, 53-58.

35. Zhu P, Tong SL, Hao JH, et al. (2015) Cord blood vitamin D and neurocognitive development are nonlinearly related in toddlers. J Nutr 145, 1232-1238. 